\title{
Ethanol at Low Concentration Attenuates Diabetes Induced Lung Injury in Rats Model
}

\author{
Jun-Feng Hu, ${ }^{1}$ Guan-Jun Zhang, ${ }^{2}$ Lei Wang, ${ }^{2}$ Pin-Fang Kang, ${ }^{3}$ Jun Li, \\ Hong-Ju Wang, ${ }^{3}$ Qin Gao, ${ }^{2}$ and Yu-Qing Chen ${ }^{1}$ \\ ${ }^{1}$ Department of Respiratory Disease, The First Affiliated Hospital of Bengbu Medical College, Bengbu, Anhui 233004, China \\ ${ }^{2}$ Department of Physiology, Bengbu Medical College, 2600 Dong Hai Avenue, Bengbu 233030, China \\ ${ }^{3}$ Department of Cardiovascular Disease, The First Affiliated Hospital of Bengbu Medical College, Bengbu, Anhui 233004, China \\ ${ }^{4}$ Dongzhimen Hospital, Beijing University of Chinese Medicine, Beijing 100700, China
}

Correspondence should be addressed to Qin Gao; bbmcgq@126.com

Received 25 April 2014; Accepted 26 May 2014; Published 12 June 2014

Academic Editor: Nikolaos Papanas

Copyright (C) 2014 Jun-Feng Hu et al. This is an open access article distributed under the Creative Commons Attribution License, which permits unrestricted use, distribution, and reproduction in any medium, provided the original work is properly cited.

\begin{abstract}
To observe the changes of lung injury when diabetic rats were treated with low concentration of ethanol (EtOH) and analyze the related mechanisms, male Sprague-Dawley (SD) rats were divided into control, diabetic (DM), and EtOH+DM groups. Diabetic rat was mimicked by injection of streptozotocin intraperitoneally. Fasting blood glucose (FBG) level, lung weight (LW), body weight (BW), and LW/BW were measured. The changes of lung tissue and Type II alveolar cell were detected. Pulmonary malondialdehyde (MDA) content and superoxide dismutase (SOD) activity were measured; meanwhile, ALDH2 mRNA and protein expressions were detected by RT-PCR and western blotting, respectively. Compared with control group, in DM group, SOD activity was decreased; FBG level, LW/BW, MDA content, ALDH2 mRNA, and protein expressions were decreased. Compared with DM group, in $\mathrm{EtOH}+\mathrm{DM}$ group, SOD activity, ALDH2 mRNA, and protein expressions were increased; LW/BW and MDA content were decreased. The structures of lung tissue and lamellar bodies were collapsed in DM group; the injury was attenuated in EtOH+DM group. Our findings suggested that, in diabetic rat, pulmonary ALDH2 expression was decreased accompanying lung injury. EtOH at low concentration decreased diabetes induced lung injury through activating ALDH2 expression.
\end{abstract}

\section{Introduction}

According to an estimation of the World Health Organization (WHO), more than 366 million people worldwide will have diabetes mellitus in 2030; the urban population in developing countries is projected to double between 2000 and 2030. Diabetes mellitus (DM) is an endocrine and metabolic disease which affects almost all organs in our body. Although the lungs are not thought to be primarily affected by diabetes and lung physiological and structural abnormalities are happening in both type 1 and type 2 diabetes, the lung should be considered as a "target organ" [1].

Chronic hyperglycemia is considered as the main reason for diabetes complications. In the process of prolonged increased glucose concentration, reactive oxygen species (ROS) overproduction may be one of the key factors which induce organ injury. Decreased antioxidative function and increased oxidative stress were seen in the lung of diabetic rats and rabbits $[2,3]$. Mitochondria dysfunction will cause oxidative stress injury which is one of the underlying factors for neurodegenerative diseases, diabetes, cardiovascular diseases, cancer, and so on [4]. Physiological hypoxia triggers the changes of mitochondrial redox and increases superoxide generation at Complex III in alveolar epithelial cells [5]. Mitochondrial aldehyde dehydrogenase 2 (ALDH2) is a nuclear-encoded mitochondrial enzyme that localizes in mitochondrial matrix [6]; ALDH2 protein had been detected in liver, lung, heart, kidney, testis, esophagus, stomach, colon, and pancreas [7]. Our previous study had reported that, in diabetic rats, ethanol (EtOH) at low concentration offered myocardial protection through activation of ALDH2 expression [8], and upregulation of ALDH2 also plays the protective effect in myocardial ischemia and reperfusion 
<smiles>CCO</smiles>

FIgURE 1: The biochemistry structure of ethanol.

injury, diabetes cardiomyopathy, and kidney and brain injury [9-12]; meanwhile, some researchers reported the association between $\mathrm{ALDH} 2$ and lung disease. $\mathrm{Xu}$ et al. reported that, in neonatal rat lung, after prolonged hyperoxic exposure, ALDH2 was downregulated. In lung epithelial cells, overexpression of ALDH2 attenuated hyperoxia-induced cell death through reduction of ROS, activation of ERK/MAPK, and PI3K-Akt signaling pathways [13]. But it remains unknown whether activation of ALDH2 expression can prevent diabetes induced lung injury. So in this study, we mimic diabetes model by intraperitoneal injection of streptozotocin (STZ) to observe the role of $\mathrm{EtOH}$ at low concentration in diabetes induced lung injury and analyze the related mechanisms.

\section{Materials and Methods}

2.1. Animals. Male Sprague-Dawley (SD) rats (200-250 g) obtained from the Animal Center of Bengbu Medical College were selected for the study. All animal studies were approved by the Animal Ethics Committee of Bengbu Medical College and performed in accordance with the ethical standards.

2.2. Chemicals and Reagents. Streptozotocin (STZ) was purchased from Sigma (St. Louis, MO, USA). Malondialdehyde (MDA) and superoxide dismutase (SOD) assay kits were from Nanjing Jiancheng Bioengineering Institute, China. Ethanol (EtOH, Figure 1) was purchased from Bengbu New Chemical Reagent Factory, China. The primers used were as follows: for ALDH2 forward: 5-GTG TTC GGA GAC GTC AAA GA$3^{\prime}$ and reverse: $5^{\prime}$-GCA GAG CTT GGG ACA GGT AA-3' and the product size was $187 \mathrm{bp}$; for $\beta$-actin forward: $5^{\prime}-\mathrm{GAT}$ GGT GGG TAT GGG TCA GAA GGA C-3' and reverse: $5^{\prime}$-GCT CAT TGC CGA TAG TGA TGA CT- $3^{\prime}$ and the product size was $630 \mathrm{bp}$. Mouse anti-ALDH 2 and anti- $\beta$-actin monoclonal antibodies were from Santa Cruz Biotechnology (CA). Goat anti-mouse secondary antibodies were purchased from Boston Co., Ltd., Wuhan, China.

2.3. Induction of Diabetes and Experimental Protocol. STZ at $55 \mathrm{mg} / \mathrm{kg}$ freshly dissolved in $0.1 \mathrm{~mol} / \mathrm{L}$ sodium citrate buffer ( $\mathrm{pH} 4.5)$ was injected intraperitoneally to induce diabetic models in overnight fasted rats. The rats in control group were injected with a similar volume of sodium citrate buffer alone. The rats with plasma fasting blood glucose level higher than $16.7 \mathrm{mmol} / \mathrm{L} 72 \mathrm{~h}$ after injection were regarded as diabetic [8]. All rats were fed for eight weeks. Animals were randomly divided into control, diabetes (DM), and $\mathrm{EtOH}+\mathrm{DM}$ groups, respectively $(n=6)$. In $\mathrm{EtOH}+\mathrm{DM}$ group, $\mathrm{DM}$ rats were fed with $2.5 \% \mathrm{EtOH}$ in their drinking water for one week to initiate drinking and then it was changed to $5 \% \mathrm{EtOH}$ continuous access through the 8 weeks.

2.4. Detection of Fasting Blood Glucose (FBG) Level, Body Weight (BW), and Lung Weight (LW). The plasma FBG level and body weight (BW) were measured at the 8th week. Lung weight (LW) was determined after the rat was sacrificed. The ratio of $\mathrm{LW} / \mathrm{BW}$ was calculated.

2.5. Detection of MDA Content and SOD Activity in Lung Tissue. At the end of the experimental period, $0.1 \mathrm{~g}$ lung tissue was homogenized in ice-cold PBS buffer. The supernatant was collected after centrifugation for $20 \mathrm{~min}(2000 \mathrm{rpm})$. The protein concentration was measured by the Bradford method. Malondialdehyde (MDA) content and superoxide dismutase (SOD) activity were detected according to the instruction manual.

2.6. Histological Observation by HE Staining. For histological analysis by light microscope, the lung tissue was harvested and fixed in $4 \%$ paraformaldehyde for 24 hours, embedded in paraffin and cut into $5 \mu \mathrm{m}$ thick serial sections, and then stained with hematoxylin and eosin (HE) for light microscope observation. Lung injury degree was evaluated according to Mikawa's scoring standards: (1) alveolar congestion, (2) hemorrhage, (3) infiltration or aggregation of neutrophils in airspace or vessel wall, and (4) thickness of alveolar wall/hyaline membrane formation. Each item was scored on a 5-point scale as follows: 0: minimal damage, 1: mild damage, 2: moderate damage, 3: severe damage, and 4: maximal damage. The final lung injury score was the summation of the four items [14].

2.7. Ultrastructure Observation of Type II Alveolar Cell by Transmission Electron Microscope. Lung tissue was dissected and small pieces were fixed with $2.5 \%$ glutaraldehyde in $0.1 \mathrm{~mol} / \mathrm{L}$ cacodylate buffer for $2 \mathrm{~h}$ and postfixed in $1 \%$ osmium tetroxide in $0.1 \mathrm{~mol} / \mathrm{L}$ cacodylate buffer for $1 \mathrm{~h}$. Ultrathin sections were cut and contrasted with uranyl acetate followed by lead citrate and observed with JEM1230 transmission electron microscope (JEOL, Japan). The changes of Type II alveolar cell were observed.

2.8. Detection of Pulmonary ALDH2 mRNA by RT-PCR. The expression of pulmonary ALDH 2 mRNA was detected by RTPCR. Briefly, total RNA was extracted with TRIzol according to the manufacturer's instructions. A total RNA ( $2 \mathrm{mg})$ were reverse transcribed to CDNA, and PCR was performed by a routine method. PCR products were analyzed on $1 \%$ agarose gel. Quantification of the result was determined through measuring the optical density of the labeled bands; the value was normalized to $\beta$-actin intensity level.

2.9. Detection of Pulmonary ALDH2 Protein Expression by Western Blot. The pulmonary ALDH2 protein expression was detected by western blot [8]. Anti-ALDH2 (1:500) antibody was used. Mouse anti- $\beta$-actin antibody $(1: 500)$ was 
TABLE 1: Changes of fasting blood glucose level (FBG), body weight (BW), lung weight (LW), lung weight/body weight (LW/BW), and lung injury score in different groups.

\begin{tabular}{lccccc}
\hline Group & FBG $(\mathrm{mmol} / \mathrm{L})$ & BW $(\mathrm{g})$ & LW $(\mathrm{g})$ & LW/BW $(\mathrm{mg} / \mathrm{g})$ & Lung injury score \\
\hline Con & $6.05 \pm 1.02$ & $443.53 \pm 23.84$ & $2.40 \pm 0.43$ & $5.39 \pm 1.94$ & $1.33 \pm 0.81$ \\
DM & $32.48 \pm 3.20^{* *}$ & $179.28 \pm 28.46^{* *}$ & $1.85 \pm 0.38^{*}$ & $10.06 \pm 1.79^{* *}$ & $9.67 \pm 1.21^{* *}$ \\
EtOH+DM & $27.15 \pm 6.67^{* * \#}$ & $243.75 \pm 11.09^{* * \# \#}$ & $1.68 \pm 0.20^{* *}$ & $6.87 \pm 0.56^{\# \#}$ & $6.33 \pm 0.82^{* * \# \#}$ \\
\hline
\end{tabular}

${ }^{*} P<0.05$ and ${ }^{* *} P<0.01$ compared with Con; ${ }^{\#} P<0.05$ and ${ }^{\# \#} P<0.01$ compared with DM.

used as an internal control. The immunoblots were exposed to X-ray film and analyzed with a digital image system.

2.10. Statistical Analysis. All results were evaluated as mean \pm S.E.M. Statistical comparisons were carried out by one-way variance analysis and the Newman-Keuls test. $P<0.05$ was considered as statistically significant.

\section{Results}

3.1. Changes of Fasting Blood Glucose Level. In contrast to control group, FBG levels in $\mathrm{DM}$ and $\mathrm{EtOH}+\mathrm{DM}$ groups were increased significantly and FBG level in $\mathrm{EtOH}+\mathrm{DM}$ group was lower than in DM group (Table 1).

3.2. Changes of Body Weight, Lung Weight, and the Ratio of Lung Weight to Body Weight. Compared with control animals, body weight (BW) and lung weight (HW) were significantly decreased in DM and $\mathrm{EtOH}+\mathrm{DM}$ groups, and LW/BW was increased in DM group. In contrast to DM group, BW was increased and LW/BW was decreased in $\mathrm{EtOH}+\mathrm{DM}$ group (Table 1).

3.3. Changes of SOD and MDA in Lung Tissues. In contrast to control rats, pulmonary SOD activity was decreased in DM group while MDA content was increased in DM and $\mathrm{EtOH}+\mathrm{DM}$ groups. In EtOH+DM group, SOD activity was higher while MDA content was lower than in DM group (Table 2).

3.4. The Histological Changes of Lung Tissue. In control group, alveolar walls in lung tissue had unique shape and size, and hemorrhage and inflammatory infiltration were rare. In DM group, alveolar septum was thickened widely, infiltration of inflammatory cells and hemorrhage appeared, and the lung injury score was higher than in control group. The injury was ameliorated in $\mathrm{EtOH}+\mathrm{DM}$ group than in DM group (Figure 2 and Table 1).

3.5. Ultrastructural Changes of Type II Alveolar Cell. In control group, many regular structure lamellar bodies appeared in Type II alveolar cell cytoplasm. In DM group, the structure of lamellar bodies was collapsed, discontinuous, and vacuolated. In EtOH+DM group, the injury degree was attenuated (Figure 3).
TABLE 2: Changes of SOD activity and MDA content in lung tissues in different groups.

\begin{tabular}{lcc}
\hline Group & SOD activity (U/mg prot.) & MDA content (nmol/prot.) \\
\hline Con & $7.55 \pm 1.94$ & $3.93 \pm 0.40$ \\
DM & $4.07 \pm 0.81^{* *}$ & $5.63 \pm 0.51^{* *}$ \\
EtOH+DM & $6.45 \pm 2.05^{\#}$ & $4.67 \pm 0.71^{* \# \#}$ \\
${ }^{*} P<0.05$ and ${ }^{* *} P<0.01$ compared with Con; ${ }^{\#} P<0.05$ and ${ }^{\# \#} P<0.01$ \\
compared with DM.
\end{tabular}

TABLE 3: Changes of ALDH2 mRNA and protein expressions in lung tissues in different groups.

\begin{tabular}{lcc}
\hline Group & ALDH2/ $\beta$-actin mRNA & ALDH2/ $\beta$-actin protein \\
\hline Con & $0.47 \pm 0.05$ & $1.17 \pm 0.19$ \\
DM & $0.35 \pm 0.04^{* *}$ & $0.85 \pm 0.10^{* *}$ \\
EtOH+DM & $0.42 \pm 0.06^{\#}$ & $1.14 \pm 0.15^{\#}$ \\
\hline
\end{tabular}

${ }^{* *} P<0.01$ compared with Con; ${ }^{\#} P<0.05$ compared with DM.

3.6. Changes of ALDH2 $m R N A$ and Protein Levels in Lung Tissue. Compared with control group, the expressions of pulmonary ALDH2 mRNA and protein were decreased in DM group; compared with DM group, pulmonary ALDH2 mRNA and protein expressions were increased in $\mathrm{ETOH}+\mathrm{DM}$ group (Figures 4 and 5 and Table 3 ).

\section{Discussion}

In the present study, we observed that lung oxidative stress injury occurred in diabetic rats, which was indicated by the increase of pulmonary MDA content and decrease of SOD activity; meanwhile, the decrease of pulmonary ALDH2 mRNA and protein expressions happened accompanying the happening of lung swelling, the destruction of pulmonary tissue, and Type II alveolar cell structure. When the diabetic rats were treated with $\mathrm{EtOH}$ at low concentration which was reported to activate ALDH2 expression $[8,10]$, lung ALDH2 mRNA and protein expressions were increased, lung oxidative stress injury and swelling degree were attenuated, the destruction of pulmonary tissue and Type II alveolar cell structure was alleviated, suggesting that downregulation of pulmonary ALDH2 was likely to be correlated with oxidative stress injury in diabetic rats, and activation of ALDH2 with $\mathrm{EtOH}$ at low concentration attenuated diabetes induced lung injury and oxidative stress overload.

In the development of diabetes, oxidative stress induced by chronic hyperglycemia plays a key role in the pathogenesis 


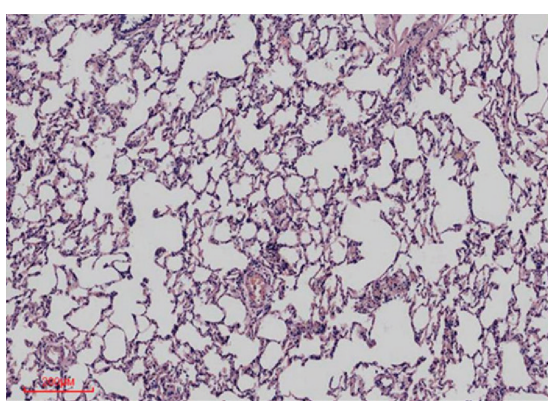

Con $(\times 100)$

(a)

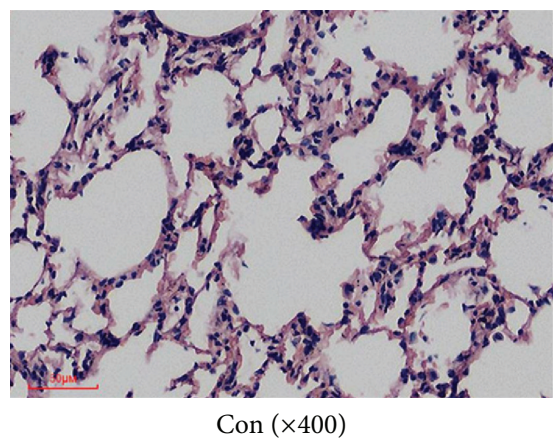

(d)

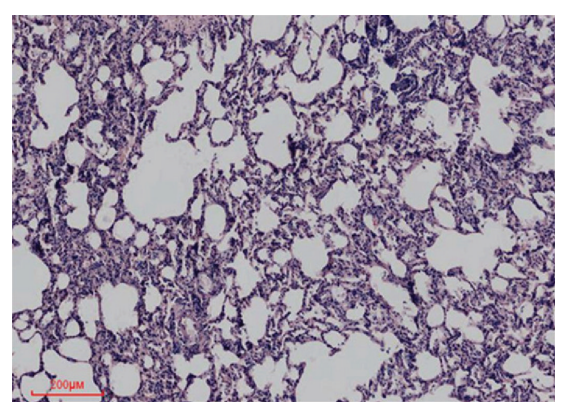

$\mathrm{DM}(\times 100)$

(b)

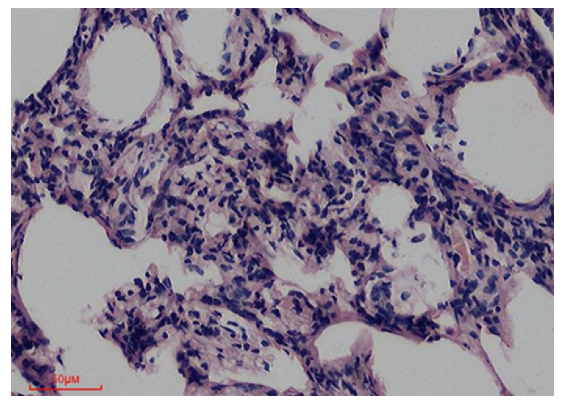

$\mathrm{DM}(\times 400)$

(e)

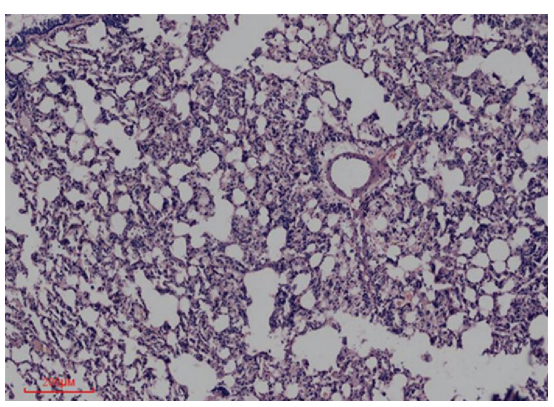

$\mathrm{EtOH}+\mathrm{DM}(\times 100)$

(c)

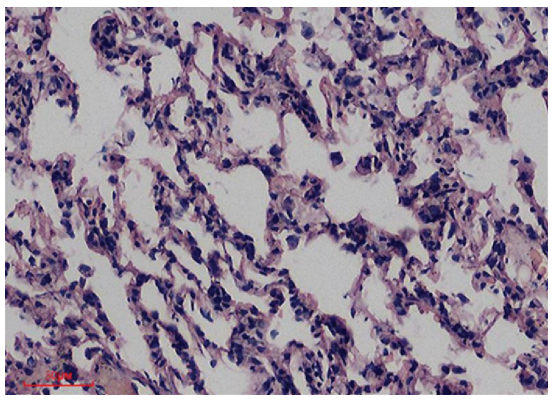

$\mathrm{EtOH}+\mathrm{DM}(\times 400)$

(f)

FIGURE 2: Histological observation of lung tissue in different groups.

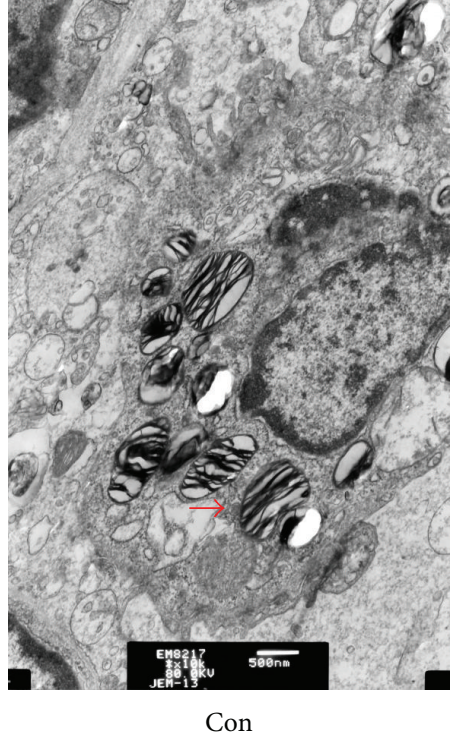

(a)

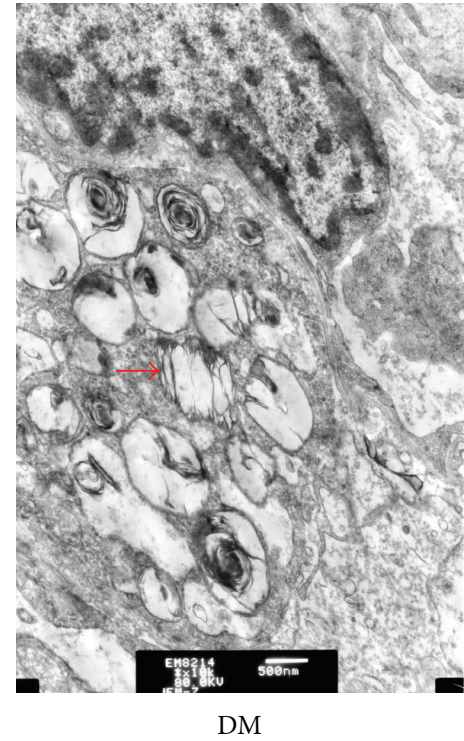

(b)

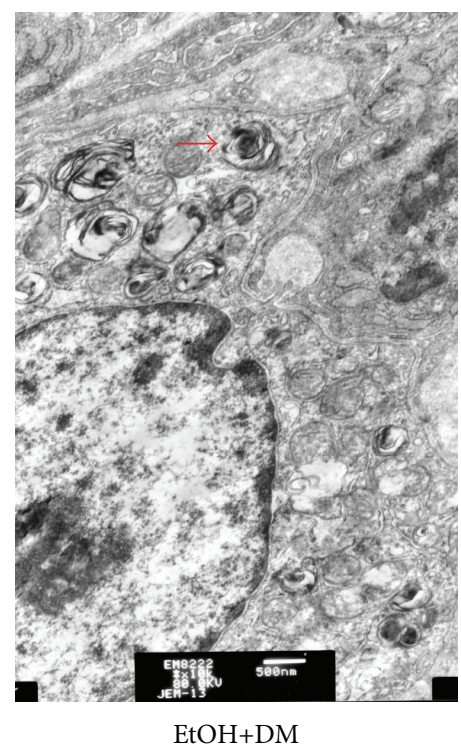

(c)

FIgURE 3: Transmission electron microscopy pictures of Type II alveolar cell in different groups ( $\times 15 \mathrm{~K})$.

of diabetes-related complications including lung diseases. Hyperglycemia can undergo autoxidation and generate oxygen radicals. Diabetes induces impairment of defense system, which is associated with reduced antioxidant capacity, abnormal activity, or expression of antioxidant enzymes [15]. An increase of MDA, a lipid peroxidation marker, accompanied by the depressing of SOD activity (one of the most important endogenous antioxidase enzymes), is assessed to imply oxidant damage. Oxidative stress can destroy the pulmonary structure. In our study, we observed that, in diabetic rat, pulmonary MDA content was increased with the decrease of SOD activity, and the structures of lung tissue and Type 


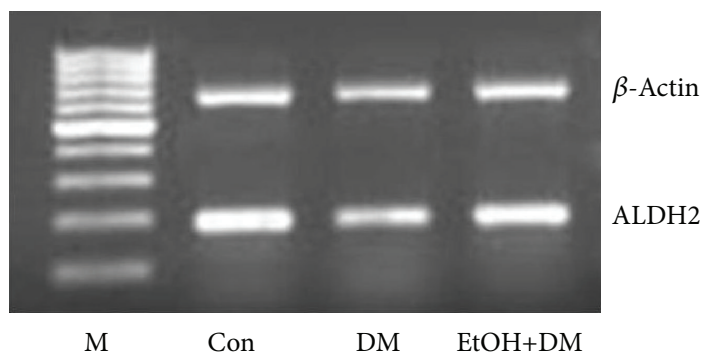

FIgURE 4: ALDH2 mRNA expression by RT-PCR.

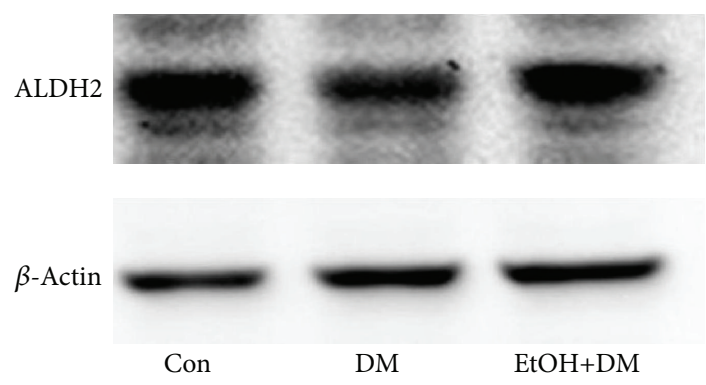

FIGURE 5: ALDH2 protein expression by western blot.

II alveolar cell were destroyed, suggesting the unbalance of lung antioxidative system induced lung injury in diabetes rats. When the diabetic rat was treated with $\mathrm{EtOH}$ at low concentration, pulmonary MDA content was decreased and SOD activity was increased accompanying the recovery of lung tissue and Type II alveolar cell structures in contrast to diabetic rat, suggesting that $\mathrm{EtOH}$ protected the lung tissue through antioxidative stress role.

ALDH2 is an enzyme that detoxifies aldehydes to carboxylic acids. The role of ALDH2 in oxidative stress had been reported by many researchers. Activation of ALDH2 with alda-1 alleviated cardiac ischemic and reperfusion injury with the decrease of 4-HNE in rodent models [8]. ALDH2 overexpression rescued neuronal survival against 4-HNE treatment in PC12 cells [16]. In male C57BL/6 mice, activation of ALDH2 with EtOH prevented renal ischemia and reperfusion injury with suppressed lipid peroxidation and increased SOD activity [12]. ALDH2 prevented ROS-induced vascular contraction in angiotensin-II induced hypertensive mice [17]. But few reports focus on the relationship of pulmonary ALDH2 and diabetes induced lung injury. ALDH2 overexpression attenuated hyperoxia-induced cell death in lung epithelial cells through reduction of ROS, activation of ERK/MAPK, and PI3K-Akt signaling pathways [13]. Since diabetes induced lung injury accompanied with oxidative stress, while increasing ALDH2 expression could decrease oxidative stress, so we investigated whether activating pulmonary ALDH2 expression by EtOH could decrease lung oxidative stress injury in diabetic rats. The results displayed pulmonary ALDH2 mRNA and protein expressions were decreased in diabetic rats, while EtOH treatment increased ALDH2 expression and, meanwhile, decreased oxidative stress injury; it suggested that, in diabetes induced lung injury, the aggravation of oxidative stress may be derived from the decrease of pulmonary ALDH2 expression and improving pulmonary ALDH2 expression could be against the happening of hyperglycemia induced oxidative stress.

It is worthwhile to note that, in our study, we selected EtOH to promote ALDH2 expression because there had been many papers reporting that $\mathrm{EtOH}$ at a suitable concentration could activate ALDH2 expression and play the protective effect $[8,10,12,18]$. But in clinic, EtOH is difficult to apply for patients because of its toxicity and addiction. So selecting an appropriate drug to activate pulmonary ALDH2 expression may be beneficial for diabetic patients who had suffered from lung injury.

In conclusion, our results indicated that, in diabetes induced lung injury in rat model, pulmonary ALDH2 expression was decreased. Treatment with EtOH at low concentration can decrease diabetes induced lung injury through activating ALDH2 expression.

\section{Conflict of Interests}

The authors have no potential conflict of interests to declare.

\section{Acknowledgments}

This work was supported by the National Natural Science Foundation of China (81000074), Foundation of Anhui Educational Committee (KJ2013B149), and Bengbu Medical College Science and Technology Development Key Foundation (Byfk12A13), China.

\section{References}

[1] M. Sandler, "Is the lung a "target organ" in diabetes mellitus?" Archives of Internal Medicine, vol. 150, no. 7, pp. 1385-1388, 1990.

[2] F. Oztay, A. Kandil, E. Gurel et al., "The relationship between nitric oxide and leptin in the lung of rat with streptozotocininduced diabetes," Cell Biochemistry and Function, vol. 26, no. 2, pp. 162-171, 2008.

[3] A. Gumieniczek, M. Krzywdziska, and M. Nowak, "Modulation of nitrosative/oxidative stress in the lung of hyperglycemic rabbits by two antidiabetics, pioglitazone and repaglinide," Experimental Lung Research, vol. 35, no. 5, pp. 371-379, 2009.

[4] J. Kang and S. Pervaiz, "Mitochondria: redox metabolism and dysfunction," Biochemistry Research International, vol. 2012, Article ID 896751, 14 pages, 2012.

[5] P. T. Schumacker, "Lung cell hypoxia: role of mitochondrial reactive oxygen species signaling in triggering responses," Proceedings of the American Thoracic Society, vol. 8, no. 6, pp. 477-484, 2011.

[6] T. Oyama, T. Isse, N. Kagawa et al., "Tissue-distribution of aldehyde dehydrogenase 2 and effects of the ALDH2 genedisruption on the expression of enzymes involved in alcohol metabolism," Frontiers in Bioscience, vol. 10, pp. 951-960, 2005.

[7] M. J. Stewart, K. Malek, and D. W. Crabb, "Distribution of messenger RNAs for aldehyde dehydrogenase 1, aldehyde dehydrogenase 2, and aldehyde dehydrogenase 5 in human tissues," Journal of Investigative Medicine, vol. 44, no. 2, pp. 4246, 1996. 
[8] Q. Gao, H.-J. Wang, X.-M. Wang et al., "Activation of ALDH2 with ethanol attenuates diabetes induced myocardial injury in rats," Food and Chemical Toxicology, vol. 56, pp. 419-424, 2013.

[9] Y. Zhang, S. A. Babcock, N. Hu, J. R. Maris, H. Wang, and J. Ren, "Mitochondrial aldehyde dehydrogenase (ALDH2) protects against streptozotocin-induced diabetic cardiomyopathy: role of GSK $3 \beta$ and mitochondrial function," BMC Medicine, vol. 10, article 40, 2012.

[10] C.-H. Chen, G. R. Budas, E. N. Churchill, M.-H. Disatnik, T. D. Hurley, and D. Mochly-Rosen, "Activation of aldehyde dehydrogenase-2 reduces ischemic damage to the heart," Science, vol. 321, no. 5895, pp. 1493-1495, 2008.

[11] J. Bai and Y. Mei, "Overexpression of aldehyde dehydrogenase-2 attenuates neurotoxicity induced by 4-hydroxynonenal in cultured primary hippocampal neurons," Neurotoxicity Research, vol. 19, no. 3, pp. 412-422, 2011.

[12] Q. Yuan, S. Hong, S. Han et al., "Preconditioning with physiological levels of ethanol protect kidney against ischemia/ reperfusion injury by modulating oxidative stress," PLoS ONE, vol. 6, no. 10, Article ID e25811, 2011.

[13] D. Xu, J. R. Guthrie, S. Mabry, T. M. Sack, and W. E. Truog, "Mitochondrial aldehyde dehydrogenase attenuates hyperoxiainduced cell death through activation of ERK/MAPK and PI3KAkt pathways in lung epithelial cells," American Journal of Physiology: Lung Cellular and Molecular Physiology, vol. 291, no. 5, pp. L966-L975, 2006.

[14] K. Mikawa, K. Nishina, Y. Takao, and H. Obara, "ONO-1714, a nitric oxide synthase inhibitor, attenuates endotoxin-induced acute lung injury in rabbits," Anesthesia and Analgesia, vol. 97, no. 6, pp. 1751-1755, 2003.

[15] V. K. Çelık, Z. D. Şahın, İ. Sari, and S. Bakir, "Comparison of oxidant/antioxidant, detoxification systems in various tissue homogenates and mitochondria of rats with diabetes induced by streptozocin," Experimental Diabetes Research, vol. 2012, Article ID 386831, 5 pages, 2012.

[16] W.-C. Lee, H.-Y. Wong, Y.-Y. Chai et al., "Lipid peroxidation dysregulation in ischemic stroke: plasma 4-HNE as a potential biomarker?" Biochemical and Biophysical Research Communications, vol. 425, no. 4, pp. 842-847, 2012.

[17] H. Choi, R. C. Tostes, and R. C. Webb, "Mitochondrial aldehyde dehydrogenase prevents ROS-induced vascular contraction in angiotensin-II hypertensive mice," Journal of the American Society of Hypertension, vol. 5, no. 3, pp. 154-160, 2011.

[18] E. N. Churchill, M.-H. Disatnik, and D. Mochly-Rosen, "Time-dependent and ethanol-induced cardiac protection from ischemia mediated by mitochondrial translocation of eopenPKC and activation of aldehyde dehydrogenase 2," Journal of Molecular and Cellular Cardiology, vol. 46, no. 2, pp. 278-284, 2009. 


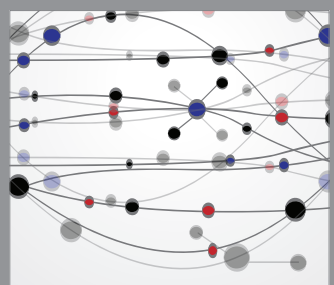

The Scientific World Journal
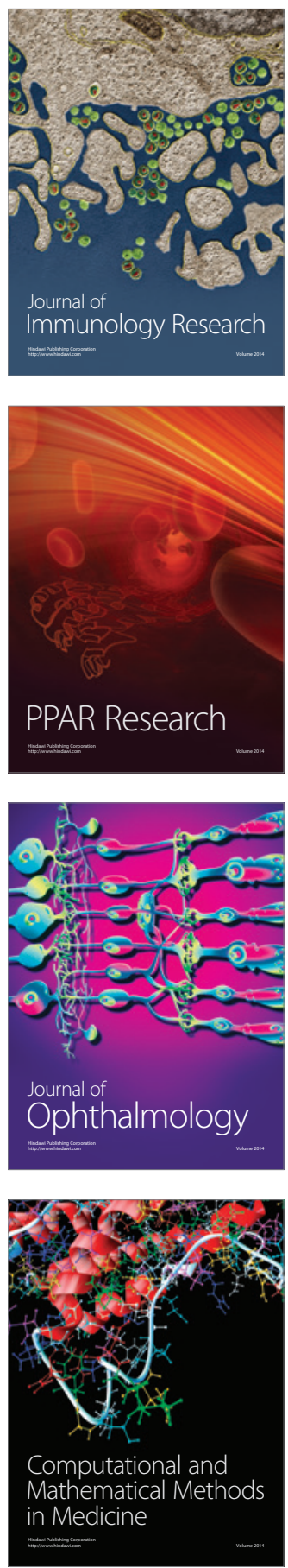

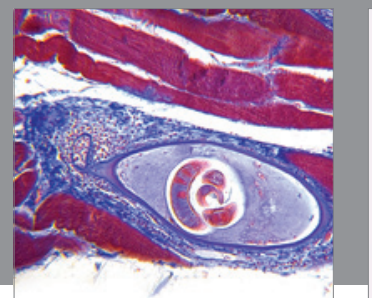

Gastroenterology

Research and Practice
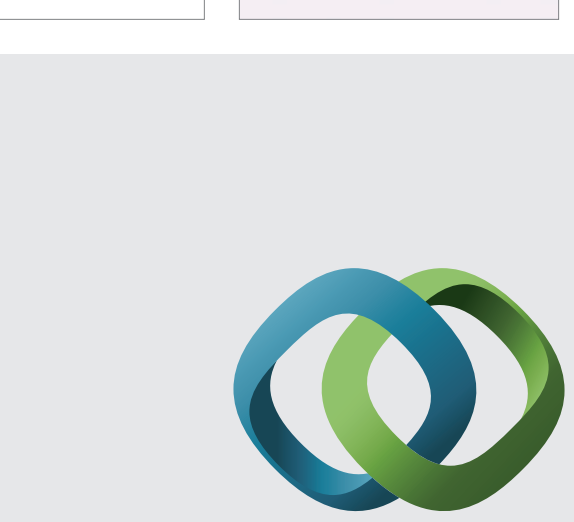

\section{Hindawi}

Submit your manuscripts at

http://www.hindawi.com
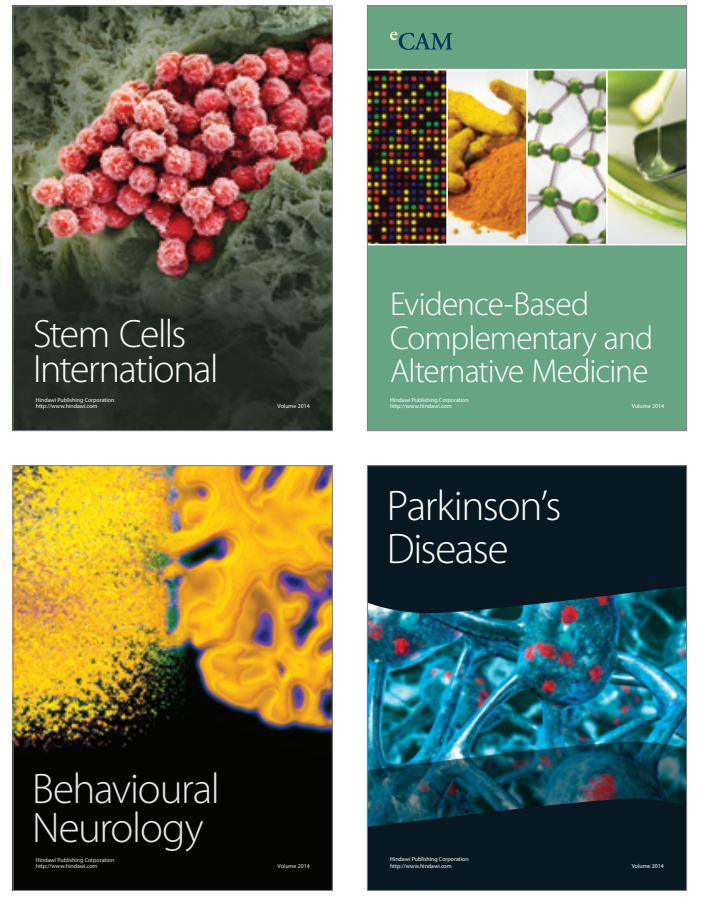
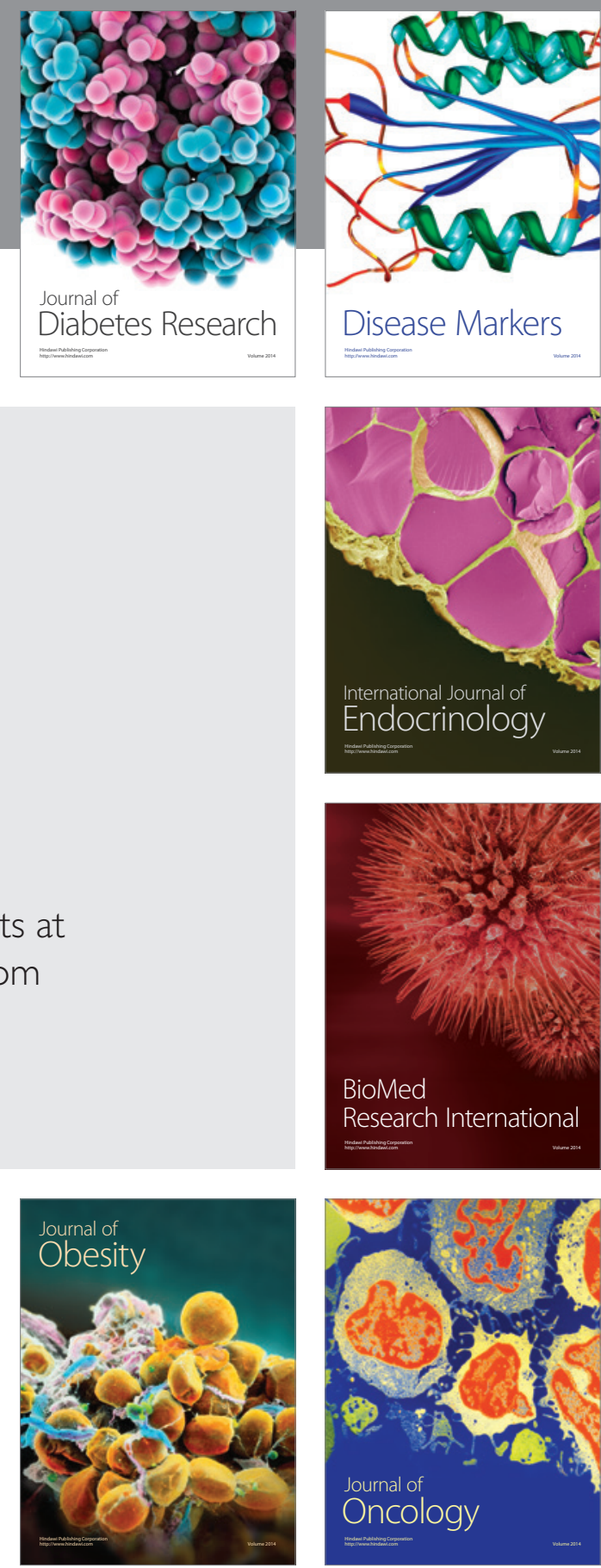

Disease Markers
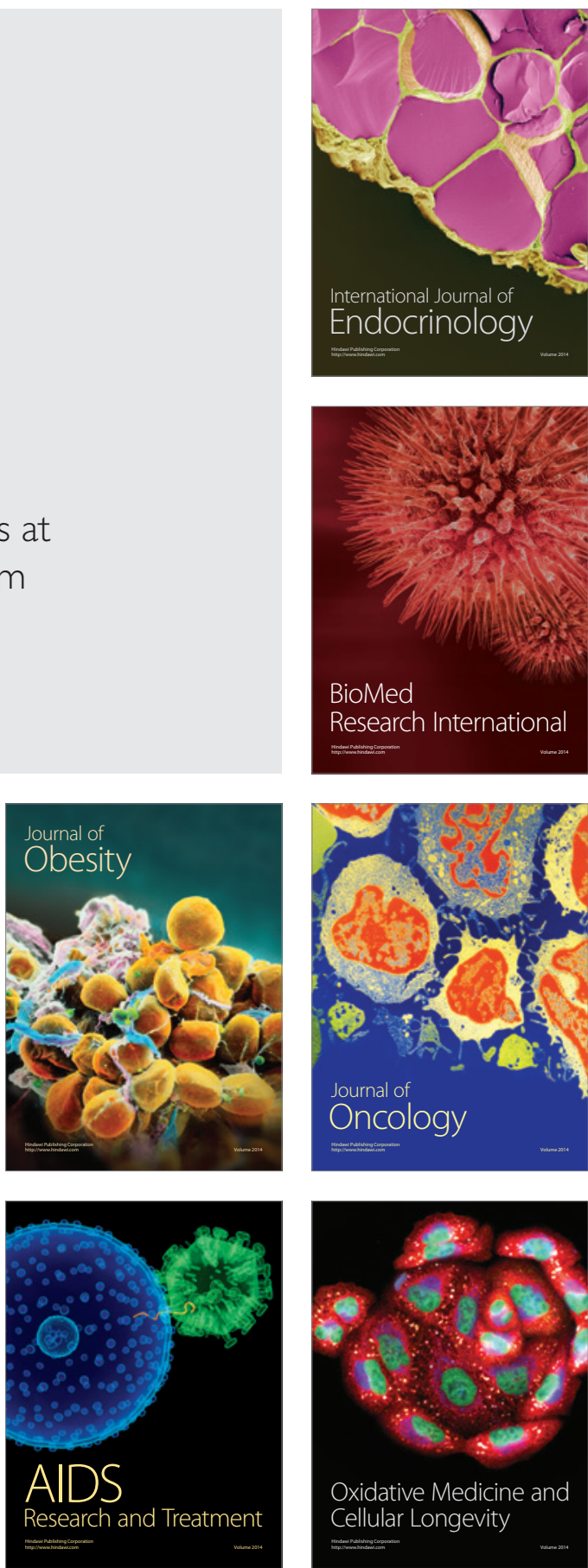